Published in final edited form as:

Top Magn Reson Imaging. 2014 February ; 23(1): 3-11. doi:10.1097/RMR.0000000000000013.

\title{
Cardiac $T_{1}$ Imaging
}

\author{
Michael Jerosch-Herold, $\mathrm{PhD}^{1}$ and Raymond Y. Kwong, MD, MPH ${ }^{2}$ \\ ${ }^{1}$ Department of Radiology, Brigham and Women's Hospital, Harvard Medical School, Boston, MA \\ 02114
}

${ }^{2}$ Cardiovascular Division, Department of Medicine, Brigham and Women's Hospital, Harvard Medical School, Boston, MA 02114

\begin{abstract}
$\mathrm{T}_{1}$ mapping of the heart has evolved into a valuable tool to evaluate myocardial tissue properties, with or without contrast injection, including assessment of myocardial edema and free water content, extra-cellular volume (expansion), and most recently cardiomyocyte hypertrophy. The MRI pulse sequence techniques developed for these applications have had to address at least two important considerations for cardiac applications: measure magnetization inversion recoveries during cardiac motion with sufficient temporal resolution for the shortest expected $\mathrm{T}_{1}$ values, and, secondly, obtain these measurements within a time during which a patient can comfortably suspend breathing. So-called Look-Locker techniques, and variants thereof, which all sample multiple points of a magnetization recovery after each magnetization preparation have therefore become a mainstay in this field. The rapid pace of advances and new findings based on cardiac $T_{1}$ mapping for assessment of diffuse fibrosis, or myocardial edema show that these techniques enrich the capabilities of MRI for myocardial tissue profiling, which is arguably unmatched by other cardiac imaging modalities.
\end{abstract}

\section{Introduction}

$\mathrm{T}_{1}$ mapping of the heart has over the last few years seen a dramatic increase in its use in cardiac magnetic resonance (CMR) imaging. This is largely due to the recognition that $\mathrm{T}_{1}$ mapping can quantify a spectrum of changes in myocardial tissue structure and composition that occur with the onset of heart diseases. In combination with the administration of an extra-cellular contrast agent, $\mathrm{T}_{1}$ mapping has proven valuable for detecting expansion of the extra-cellular space, which reflects the build-up of fibrosis in the myocardium. This is complementary to late-gadolinium enhancement (LGE) imaging, which is a widely applied clinical CMR technique that visualizes myocardial scar and myocardial necrosis. The reliance in LGE imaging on detecting differences in delayed contrast enhancement within the ventricular walls, renders it difficult to detect diffuse myocardial fibrosis that may be generalized throughout the entire heart. The increasing use of $\mathrm{T}_{1}$ mapping was initially, to a large degree, motivated by this perceived short-coming of LGE imaging and the need to detect more "subtle" forms of myocardial fibrosis.

Correspondence to: Michael Jerosch-Herold PhD Associate Professor Department of Radiology, Brigham and Women's Hospital 75 Francis Street, Radiology BWH Box \#22 Boston, MA 02115 Tel: (617) 525-8959 Fax: (617) 264-5245 mjeroch-herold@ partners.org. 
In 2004, a study in patients with aortic regurgitation provided an important impetus for developing $\mathrm{T}_{1}$ mapping to detect diffuse myocardial fibrosis. ${ }^{1}$ Post-contrast $\mathrm{T}_{1}$ values, acquired at a fixed time after contrast injection, were significantly shorter in patients with aortic regurgitation than in healthy volunteers. This finding suggested that the distribution volume for the extra-cellular contrast agent was larger than in normal myocardium, possibly due to the build-up of connective tissue within the interstitial space. Previous studies of endo-myocardial biopsy samples in patients with aortic regurgitation had documented a pervasive burden of diffuse interstitial fibrosis. ${ }^{2,3}$

One of the main applications emerging from cardiac $\mathrm{T}_{1}$ imaging is the determination of the extra-cellular volume (ECV) fraction, ${ }^{4,5}$ using pre- and post-contrast $\mathrm{T}_{1}$ measurements. It has been shown that ECV is a good surrogate marker of interstitial fibrosis in diseases like aortic stenosis, ${ }^{6}$ hypertrophic cardiomyopathy (HCM),${ }^{5}$ and dilated cardiomyopathy (DCM). ${ }^{4}$ More recently, non-contrast $\mathrm{T}_{1}$ mapping has been proven to yield high diagnostic accuracy for the detection of changes in free water content occurring with acute edema. ${ }^{7,8}$ These newer applications may pave the way for an improved quantification of the area-atrisk in ischemic heart disease, and the diagnosis of myocarditis. Abnormal native (i.e. without contrast) $T_{1}$ values were also reported for patients with hypertrophic and dilated cardiomyopathies, ${ }^{9}$ though the origin of these $\mathrm{T}_{1}$ changes remain under investigation. This review of cardiac $T_{1}$ imaging will start with an introduction of the CMR techniques used for $\mathrm{T}_{1}$ measurements, and then proceed to the post-processing and interpretation of the data.

\section{Pulse Sequence Techniques for $T_{1}$ Imaging}

Measuring $T_{1}$ has been an important focus of MRI investigators since the beginnings of this imaging modality, and providing the impetus for the development of multiple approaches for rapid $\mathrm{T}_{1}$ imaging and quantification. $\mathrm{T}_{1}$ is the characteristic time constant with which longitudinal magnetization recovers after being inverted with a radio-frequency pulse. In blood and tissue the recovery of the longitudinal magnetization after inversion can in many cases be described by a single exponential function, and $T_{1}$ then refers to the time constant in the exponential. If $M_{Z}(T I)$ denotes the longitudinal magnetization as a function of $T I$, the time after inversion, one can express a single-exponential inversion recovery as:

$$
M_{z}(T I)=M_{0}\left[1-I N V \cdot \exp \left(-T I / T_{1}\right)\right]
$$

where $M_{O}$ is the equilibrium magnetization before inversion, and $I N V$ the inversion factor. For a perfect inversion we have $I N V=2$, which yields $M_{Z}(T I=0)=-M_{o}$, i.e. right after the inversion the longitudinal magnetization has a value that is the negative of its value before the inversion pulse. The measurement of $T_{1}$ always involves disturbing the longitudinal magnetization from its equilibrium, and then determining the rate at which the longitudinal magnetization recovers. A simple $T_{1}$ experiment could take the form of inversion of the magnetization, followed a time $\mathrm{t}=\mathrm{TI}$ later, by sampling of the longitudinal magnetization with a radio-frequency "read-out " pulse. This process of inversion and read-out has to be repeated for a series of TI values - at least 2-3 TI values in the case of a single exponential as described by equation 1 . The sampled signal-intensities can then be fit to equation 1 , to

Top Magn Reson Imaging. Author manuscript; available in PMC 2017 February 14. 
determine $T_{1}$, assuming that a single exponential represents an adequate description of the inversion recovery. It should be noted that between repetition of each inversion and read-out, sufficient time should elapse so that the longitudinal magnetization can return to its $M_{O}$ equilibrium value. If this is not the case, one has to explicitly take into account the incomplete magnetization recovery between inversion pulses.

Before introducing MRI techniques for rapid $\mathrm{T}_{1}$ imaging, it is worthwhile to first describe the gold-standard for T1 measurements, which we will refer to here, as the inversionrecovery-prepared single spin-echo (IR-SE) technique, illustrated in Figure 1. With this technique one inverts the magnetization, and samples the longitudinal magnetization at a specific time after the inversion (known as inversion time, TI), with a single echo read-out. It is referred to as a single point technique, because the longitudinal magnetization is sampled for only one TI value after each magnetization inversion. As mentioned before, the experiment is repeated for a series of TI's, in addition to the repetitions required for all the image encoding steps, i.e. for a $2 \mathrm{D} \mathrm{T}$ map the total number of steps is (number of TI's) * (number of phase-encoding steps). These steps should be repeated with a delay time of 4-5 times the expected $\mathrm{T}_{1}$, to allow for nearly complete relaxation recovery after each inversion pulse. With $\mathrm{T}_{1}$ 's of normal myocardium and blood on the order of 1-2 seconds, this would clearly result in prohibitive image acquisition times, rendering in-vivo T1 mapping with this technique impractical. $T_{1}$ measurements with the IR-SE technique have therefore been relegated to measurements on phantoms, to validate other more rapid techniques.

\section{Look-Locker $\mathrm{T}_{1}$ Imaging}

The requirement of complete magnetization recovery between magnetization inversions can render $\mathrm{T}_{1}$ imaging very time-consuming. One way to overcome this limitation is to sample the longitudinal magnetization for multiple TI values after an inversion pulse. For sampling of the magnetization after its inversion one could use a train of low-flip angle radiofrequency $(\mathrm{RF})$ pulses, and sample the transverse magnetization after each RF pulse (e.g. by producing a gradient echo).

Look and Locker in a landmark publication from $1970,{ }^{10}$ well before the introduction of MRI, described such a technique, which in one form used a periodic train of constant flipangle radio-frequency (RF) pulses, and sampling of the MR signal after each RF pulse. The RF pulse train, if sufficiently long, drives the magnetization to a "steady state". The steadystate value of the longitudinal magnetization is lower than the value corresponding to the undisturbed equilibrium $\left(M_{O}\right)$. The rate of change of the signal amplitudes sampled with the periodic pulse train can be used to derive $\mathrm{T}_{1}$, without prior calibration of the RF flip angle.

If we call the signal right after the first RF pulse $S(O)$, and the signal in the equilibrium state as $S(\infty)$, one can define a "dynamic range" $(D R)$ for the Look-Locker experiment as the difference $D R=S(0)-S(\infty) .{ }^{11}$ It can be shown that the error in the estimation of $\mathrm{T}_{1}$ can be reduced by maximizing the dynamic range. For the purpose of maximizing the dynamic range, the Look-Locker train of RF pulses can be preceded by a magnetization-inversion pulse, giving rise to what can be described as multi-point sampling of the inversion recovery. Sampling of the longitudinal magnetization generally results in a disturbance of the magnetization recovery, and this disturbance grows in magnitude with the flip angle of the 
excitation pulse. The effect of the RF pulses is to drive the magnetization to a steady-state equilibrium level that is below the signal equilibrium in an undisturbed inversion recovery, and to speed up the rate at which this state-state equilibrium is reached, with the rate increasing with increasing RF flip angle, as illustrated in Figure 2. This is in particular true for gradient echo sequences without steady-state free precession, i.e. pulse sequences where the transverse magnetization is spoiled and not returned to the z-axis by the next RF pulse in the train. The effective $\mathrm{T}_{1}$ during a Look-Locker multi-point read-out, often denoted by $\mathrm{T}_{1} *$, is therefore not equal to the $\mathrm{T}_{1}$ that would be measured from a inversion recovery that remains undisturbed until the acquisition of a single sample. Nevertheless $\mathrm{T}_{1} *$ can be used to estimate the true $\mathrm{T}_{1}$, by taking into account the specifics of the sampling of the inversion recovery.

For a Look-Locker multi-point read-out, the magnetization (or sampled signal) relaxes towards a "steady-state" $M_{Z}(\infty)$ (or $S(\infty)$ ) with an apparent, or effective $\mathrm{T}_{1}$ given by:

$$
T_{1}^{*}=T R /\left(T R / T_{1}-\ln (\cos (\alpha)) .\right.
$$

TR is the time between RF pulses, and a the (constant) flip angle of the RF pulses. Figure 3 illustrates how the effective $T_{1}$ becomes insensitive to the true $T_{1}$ when the flip angles of the RF pulse train increases. This means that the flip angle of the RF "read-out" pulses should be kept relatively low $\left(\sim 5-15^{\circ}\right)$ for $\mathrm{T}_{1}$ measurements.

The Look-Locker scheme was initially introduced for pulsed NMR experiments without any gradient pulses. The adaptation to imaging studies was relatively straightforward, and involved introducing gradient waveforms and an additional loop in the pulse sequence for the spatial phase-encoding steps. ${ }^{12}$ The imaging version of the Look-Locker technique is also known by the TOMROP (T One by Multiple Read Out Pulses) acronym. ${ }^{13}, 14$ The addition of the gradient pulses for slice selection and spatial encoding has no direct effect on the inversion recovery

An additional speed-up can be achieved by acquiring segments of phase-encoded lines of data, instead of just a single-phase encoded line. The segment size determines the TI resolution that one achieves.

For cardiac applications, the start of each Look-Locker cycle is electrocardiographically triggered. As data are acquired over the entire cardiac cycle, the images resemble a cardiac cine loop, with the important distinction that each frame corresponds to a different TI time. Figure 4 shows the sequence diagram for a Look-Locker acquisition.

\section{Modified Look-Locker $\mathrm{T}_{1}$ Imaging (MOLLI)}

The Look-Locker technique with gradient-echo read-outs for multiple TI values has the disadvantage that the inversion recovery is altered by the application of the RF pulses. In the worst case this can result in poor sensitivity to $\mathrm{T}_{1}$ differences. The effect of the RF pulses and spoiling of transverse magnetization on the inversion recovery can be reduced by using a steady-state free precession (SSFP) technique for image read-out, as shown in 2001 by 
Scheffler and Henning, ${ }^{15}$ setting the stage for a heightened interest in SSFP techniques for $\mathrm{T}_{1}$ measurements.

The MOLLI technique uses SSFP image-read-outs and was introduced for cardiac $\mathrm{T}_{1}$ mapping so that the image acquisition is performed at the same time point in the cardiac cycle for all TI's. ${ }^{16,17}$ In addition, several Look-Locker cycles are merged into one breathhold to avoid breathing related mis-registration of the images for different TI's. This renders it feasible to follow inversion-recoveries at the pixel level.

Each Look-Locker cycle starts with an inversion pulse, followed by rapid single-shot acquisition of images with steady-state free precession at a fixed, user-defined point in the cardiac cycle, typically diastole. For subsequent Look-Locker cycles the inversion pulse is shifted within the cardiac cycle by $\sim 50-300 \mathrm{~ms}$, but the timing of the image SSFP image acquisition remains constant. Each Look-Locker cycle yields images with TI increments corresponding to the heart beat duration, while variation of the timing for the inversion pulse gives TI times with smaller 100-200 ms increments, relative to the already acquired points. The end result is a series of images with TI times covering typically a TI range up to 2-5 heart beats. Figure 5 illustrates a scheme for the MOLLI acquisition. After the first LookLocker cycle in Figure 5, there is a relaxation period extending over four heart beats to allow complete recovery before application of an inversion pulse for the next Look-Locker cycle.

The "single-shot" SSFP image acquisitions start with 5-10 dummy TR cycles using excitation pulses with linearly increasing startup angles (LISA) to transition to a steady-state of free precession. Partial Fourier encoding can be used with a linear phase-encoding order to reach the center of k-space quickly. E.g. approximately only 30 phase-encodings are used to reach the center of k-space, with full sampling of the conjugate portion of k-space after traversing the center. The partial Fourier encoding allows sampling closer to the beginning of the IR.

MOLLI acquisition schemes are summarized in terms of the number of heart beats that are used for acquiring images after each inversion pulse. For example a "5-3-3" scheme would mean that 5 images are acquired after the first inversion pulse in the first Look-Locker cycle. In the next two cycles 3 image are acquired after the inversion pulse. If the patient's heart rate is $60 \mathrm{bpm}$, and the TI shift between Look-Locker cycles is $150 \mathrm{~ms}$, then one would obtain approximately the following $10 \mathrm{TI}$ times, listed here in the order of acquisition: 100, 1100, 2100, 3800, 4800, 200, 1200, 2200, 300, 1300, $2300 \mathrm{~ms}$.

An increasingly more often used variant of MOLLI, uses a 3 cycle MOLLI scheme, with abbreviated recovery times in the $2^{\text {nd }}$ and final LL cycle, to shorten the time required for breath-holding. ${ }^{18}$ This shortened MOLLI (shMOLLI) method for $\mathrm{T}_{1}$ mapping, illustrated in Figure 6, requires conditional post-processing of the data, because only when $T_{1}$ is sufficiently short to achieve an approximately complete recovery in the previous LL cycle is a data sample from the next LL cycle used for the $\mathrm{T}_{1}$ determination. ${ }^{18}$ 


\section{Saturation-Recovery Prepared $\mathrm{T}_{\mathbf{1}}$ Mapping (SASHA)}

With SASHA (Saturation-recovery single-Shot Acquisition), ${ }^{19} \mathrm{~T}_{1}$ mapping is also, like with MOLLI and shMOLLI, performed in a single breath-hold, starting with the acquisition of a equilibrium (or "no-saturation") image without any magnetization preparation. This is followed by Look-Locker cycles, using a saturation-recovery preparation. The first image defines the signal intensity in the equilibrium state, i.e. the signal intensity from completely relaxed magnetization, denoted here by $M_{O}$. All subsequent images are acquired after a magnetization saturation pulse with varying times after saturation (TS). The variation of regional signal intensity is in most instances well approximated by a single-exponential recovery, described by the following equation:

$$
M_{z}(T S)=M_{0} \cdot\left[1-\exp \left(-T S / T_{1}\right]\right.
$$

Compared to an inversion-pulse preparation, the dynamic range of SASHA is only half as large, which in principle implies a larger uncertainty (or error) for the estimation of $\mathrm{T}_{1}$. The use of a saturation-recovery preparations renders the measured signal intensities heart rate insensitive, thereby giving SASHA an important practical advantage for cardiac T1 measurements, over MOLLI.

Table 1 provides a summary of important characteristics and advantages of techniques for $\mathrm{T}_{1}$ quantification included in this review.

\section{Inversion-Recovery Analysis and Post-Processing}

The analytical expression for an inversion or saturation recovery with a single exponential dependence on the time after inversion (or saturation), described by equation 1 , can be rewritten in terms of measured signal intensities ( $S$, in arbitrary units) as:

$$
S(T I)=S_{0} \cdot\left[1-I N V \exp \left(-T I / T_{1}\right]\right.
$$

where $\mathrm{S}_{\mathrm{O}}$ is the equilibrium signal, and $I N V$, the inversion factor, with $I N V=2$ for a complete inversion, and $I N V=1$ for a saturation preparation. In most cases the measurements are made from signal intensity images for a fixed pixel location, or a user-defined region of interest and the inversion recovery is then presented in terms of magnitude data. For a saturation recovery any loss of polarity information is immaterial.

A non-linear least squares algorithm, or the downhill simplex optimization algorithm have been used to estimate $T_{1}$, by minimization of the residual differences between fit and measured data points. In principle it is possible to specify an inversion-recovery function for the magnitude signal as target for the non-linear least squares fitting. The resulting $\mathrm{T}_{1}$ values will be the same as obtained from a fit with the above equation 4, after restoring the phase (sign) of the signal. But phase (polarity) restoration makes fitting more reliable, in particular if the number of TI samples is relatively low (e.g. 5-10). In the case of MOLLI or shMOLLI IR fits it is therefore advisable to restore the polarity of the IR signal.

Top Magn Reson Imaging. Author manuscript; available in PMC 2017 February 14. 
Phase-restoration can take several forms: a phase reference image can be acquired at the end of the inversion recovery to calculate phase-sensitive inversion recovery signal values for each TI. Alternatively, the phase can be restored after data acquisition (without phase reference data) from the magnitude IR curve: either the user identifies the location of the zero-crossing for the IR curve, or this is done iteratively, for all possible locations of the zero-crossing, by fitting in each case the corresponding polarity-restored IR curve to equation 4 . The optimal zero-crossing point can then be identified by the fit with the lowest value for the sum of squared residuals (chi-square). Figure 7 illustrates this latter approach.

For Look-Locker (or TOMROP) acquisitions with a gradient echo technique, the $\mathrm{T}_{1}$ obtained from fitting the IR curve gives an effective $\mathrm{T}_{1}$, called $\mathrm{T}_{1}{ }^{*}$, that includes the effects of the RF pulses. Equation 2 showed how $\mathrm{T}_{1}$ * varies with TR and the RF flip angle. As the RF pulse flip-angle varies spatially and is generally only determined so that its mean for a slice has a certain predefined value, this equation is of limited value to obtain for a specific region of interest or a pixel the true $\mathrm{T}_{1}$ from $\mathrm{T}_{1}$ *. Instead the effect of the RF pulses can be estimated from the ratio of the undisturbed magnetization $\left(\mathrm{M}_{\mathrm{o}}\right.$, or its absolute value right after complete inversion), and the steady state magnetization reached during application of the RF pulse train $\left(M_{\infty}\right)$. This steady state value is given by:

$$
M_{\infty}=M_{0} \frac{1-E_{1}}{1-E_{1}^{*}},
$$

where $E_{1}=\exp \left(-T R / T_{1}\right)$, and $E_{1} *=\exp \left(-T R / T_{1} *\right), T_{1}$ is the true $\mathrm{T}_{1}, \mathrm{~T}_{1} *$ the effective $\mathrm{T}_{1}$ during the Look-Locker cycle, and TR the repetition time for the gradient echo sequence. If the measured inversion recovery is fit with the three parameter function $S(T I)=A+B$. $\exp \left(-T I / T_{1} *\right)$, then A corresponds to $M_{\infty}$ in equation 5 , and B corresponds to $-\left(M_{0}+M_{\infty}\right)$ $\mathrm{T}_{1}$ he true $\mathrm{T}_{1}$ can be written as: ${ }^{20}$

$$
T_{1}=\frac{-T R}{\ln \left[1-\frac{1-E_{1}^{*}}{B / A-1}\right]} \approx \frac{T_{1}^{*}}{B / A-1} .
$$

In equation 6 it is assumed that the fully relaxed magnetization $\left(M_{O}\right)$ was completely inverted at the beginning of the Look-Locker cycle.

The approximation on the right hand side applies when $\exp \left(-T R / T_{1} *\right)<1$, which is reasonable when TR is on the order of a few $\mathrm{ms}$, and $\mathrm{T}_{1} *$ on the order of $100 \mathrm{~ms}$ or longer. This right hand side of eq. 6 is identical to the result derived by Deichmann and Haase, ${ }^{21}$ and is used in an open-source software package for $\mathrm{T}_{1}$ mapping. ${ }^{22}$ Interestingly the right hand side of equation 6 is also sometimes used to "correct" the $T_{1}$ measured with MOLLI sequences, though this appears to be an "empirical" correction for the "imperfections" in SSFP read-outs, like off-resonance shifts. ${ }^{17}$

An important advantage of MOLLI and SASHA is that all images are acquired in the same cardiac phase. For post-processing, and assuming a regular heart rhythm of the subject, a

Top Magn Reson Imaging. Author manuscript; available in PMC 2017 February 14. 
user needs to segment the myocardium in only one image, and can then automatically propagate the contours to all other images. Furthermore, $\mathrm{T}_{1}$ maps can in principle be generated with pixel-level resolution. With Look-Locker techniques where images are acquired over nearly the entire cardiac cycle, pixel level maps can only be produced by tracking pixel locations over the cardiac cycle. An example is shown in Figure 8.

\section{Parameters Derived from Cardiac $\mathrm{T}_{1}$ Measurements: Partition Coefficient and Extra- Cellular Volume Fraction}

In myocardial tissue and blood the inverse of $T_{1}$, the relaxation rate constant $R_{1}\left(=1 / T_{1}\right)$, changes proportionally to contrast agent concentration, at least when $R_{1}$ is not increased above 2-3 s-1 by contrast administration. ${ }^{23}$ For our initial considerations we therefore assume the following relationship between $\mathrm{R}_{1}$ and contrast concentration:

$$
R_{1}=R_{10}+r_{1} \cdot[G d]
$$

where $R_{10}$ is the native (i.e. pre-contrast enhancement) $R_{1}, r_{1}$ the relaxivity of the contrast agent, and [Gd] the concentration of a gadolinium-based contrast agent. If we now assume that the contrast agent distributes in myocardial tissue only within the extra-cellular (EC) space and has there a concentration of $[G d]_{E C}$, then the above equation can be rewritten as:

$$
R_{1}(\text { tissue })=R_{10}(\text { tissue })+r_{1} \cdot[G d]_{E C} \cdot E C V
$$

where ECV stands for extra-cellular volume fraction. Similarly for blood, where gadolinium contrast is excluded from the red-blood cells, one can write:

$$
R_{1}(\text { blood })=R_{10}(\text { blood })+r_{1} \cdot[G d]_{\text {plasma }} \cdot(1-H c t),
$$

where Hct is the blood hematocrit, (1-Hct) represents the plasma volume within which the contrast can distribute, and $[\mathrm{Gd}]_{\text {plasma }}$ is the concentration of gadolinium contrast within the blood plasma. Use was made of the relation $[\mathrm{Gd}]_{\text {plasma }}=(1-\mathrm{Hct}) \cdot[\mathrm{Gd}]_{\mathrm{b}}$, which reflects the exclusion of Gd contrast from the red blood cells. If one could achieve equal concentrations of contrast within the blood plasma, and the interstitial space, i.e. $[\mathrm{Gd}]_{\mathrm{EC}}=[\mathrm{Gd}]_{\text {plasma }}$, by allowing contrast concentration to equilibrate, then the ratio for the pre-to-post-contrast change of $R,\left(R_{1}-R_{10}\right)=\Delta R_{1}$, measured in blood and tissue, respectively, gives the so-called myocardial partition coefficient for contrast, $\lambda$, which is independent of Gd concentrations. Using the superscripts ' $b$ ', and ' $t$ ', to denote whether $R_{1}$ is measured in blood and tissue, respectively, the myocardial partition coefficient for gadolinium contrast $\left(\lambda_{\mathrm{Gd}}\right)$ can be expressed as:

$$
\frac{R_{1}^{t}-R_{10}^{t}}{R_{1}^{b}-R_{10}^{b}}=\frac{\Delta R_{1}^{t}}{\Delta R_{1}^{b}}=\frac{E C V}{(1-H c t)} \equiv \lambda_{G d} .
$$


Equation 10 suggests a prescription for determining ECV. $R_{1}$ is measured in blood and tissue before and after contrast administration, and in combination with the patient's blood hematocrit one can estimate ECV. An important advantage of ECV, over using post-contrast $\mathrm{T}_{1}$ measurements, is that ECV is independent of several of the potentially confounding factors that can change $\mathrm{T}_{1}$, like the rate of clearance of contrast from blood and tissue, which depends on renal function, and may vary between patients.

\section{Interpretation of Myocardial $\mathrm{T}_{\mathbf{1}}$ Measurements}

CMR techniques for T1 quantification and mapping have achieved significant efficiency for shortening acquisition times, albeit at the expense of having to use more complex techniques than the "single-point" spin-echo technique. The latter is characterized by prohibitive acquisition times, but has the distinction of providing what is arguably the closest measure of the "true" $\mathrm{T}_{1}$.

With SSFP image read-outs, as used in MOLLI and SASHA, there may be multiple factors that can bias the $\mathrm{T}_{1}$ estimate, such as the ratio of $\mathrm{T}_{1} / \mathrm{T}_{2},{ }^{24}$ or magnetization transfer effects. ${ }^{25}$ It was initially thought that SFFP techniques may be ideal for measuring inversion recoveries, and quantifying $T_{1}$, because in contrast to other gradient echo techniques, the transverse magnetization is not spoiled, but converted to longitudinal magnetization at the end of each TR cycle, resulting in minimal disturbance to the inversion recovery. ${ }^{15}$ SFFP imaging produces a contrast dependent on the ratio of $\mathrm{T} 2$ and $\mathrm{T} 1$, while for gradient echoimaging with similarly short echo-times, the signal is predominantly $\mathrm{T} 1$-weighted.

Schmitt et al ${ }^{24}$ considered off-resonance effects and the ratio of $T_{2} / T_{1}$ on the apparent $T_{1}$ measured with bSFFP techniques, assuming a constant read-out of echoes during the inversion recovery. The magnitude of $\mathrm{T}_{1}$ errors from use of the apparent $\mathrm{T}_{1}$ was $\sim 40 \%$ for $\mathrm{T}_{2} / \mathrm{T}_{1}=0.1$, using a flip-angle of $45^{\circ}$ for the bSSFP read-outs. The error of $\mathrm{T}_{1}$ was $<10 \%$ for an off-resonance angle of $\pi / 2$. For myocardial tissue, and before administering any contrast $\mathrm{T}_{1} \sim 1150$, and $\mathrm{T}_{2} \sim 40 \mathrm{~ms}$ at 3T, i.e. $\mathrm{T}_{2} \ll<\mathrm{T}_{1}$. Though the findings by Schmitt et al would appear to be of relevance for the MOLLI technique, which uses SSFP for the image readout, it should be noted that with MOLLI only a few short SSFP readouts take place during the inversion recovery, and are spaced apart by a time given by the heartbeat duration. Kellman et al have recently showed for MOLLI that the $\mathrm{T}_{1}$ error from off-resonance effects is larger for longer $\mathrm{T}_{1}$ times, approaching $-7 \%$ for an off-resonance shift of $\pm 50 \mathrm{~Hz}$ for $\mathrm{T}_{1}=1200 \mathrm{~ms}$, and $\sim-4 \%$ for $\mathrm{T}_{1}=600 \mathrm{~ms}^{26}$

Robson et $\mathrm{al}^{25}$ have simulated the effects of magnetization transfer on $\mathrm{T}_{1}$ measurements with MOLLI, and found that MT can cause a significant bias for $\mathrm{T}_{1}$. The magnetization transfer was assumed to take place between a "dark pool" of $1 \mathrm{H}$ nuclei and mobile $1 \mathrm{H}$ nuclei from water and lipids. Potential components of this dark pool are $1 \mathrm{H}$ nuclei whose signal is otherwise not directly detected by the MRI sequences with echo-times on the order of ms, such as $1 \mathrm{H}$ in collagen. The MT effect grows in magnitude as one uses higher levels of radio-frequency power for excitation pulses, and for this reason one would expect that MT effects would be more pronounced with MOLLI measurements, than with GRE LookLocker measurements. For the latter one typically uses flip angles on the order of $10^{\circ}$, while for MOLLI flip angles on the order of $45^{\circ}$ or higher are common. 
Nacif and colleagues ${ }^{27}$ compared pre-contrast global $\mathrm{T}_{1}$ values measured at 1.5 Tesla by the Look-Locker and by MOLLI technique (mean, $1004.9 \mathrm{~ms} \pm 120.3$ versus $1034.1 \mathrm{~ms} \pm 53.1$, respectively, $\mathrm{P}=0.26$ ), and using in both cases SSFP for the image acquisition. It remains to be seen if a Look-Locker implementation with gradient-echo read-outs (without SFFP) will give a significantly higher $\mathrm{T}_{1}$, than MOLLI.

The increasing awareness of effects from MT, off-resonance shifts, and $\mathrm{T}_{2}$ relaxation on $\mathrm{T}_{1}$ measurements has raised the question of whether one can measure a "true" $\mathrm{T}_{1}$. Based on current evidence one can say that the $\mathrm{T}_{1}$ measured with a single point technique like IRprepared spin-echo imaging should give in principle a relatively accurate measure of the $T_{1}$ estimate of the mobile $1 \mathrm{H}$ species in myocardial tissue, as the IR is not disturbed by the image read-out, which are the source of MT and $\mathrm{T}_{2}$ effects. A Look-Locker read-out with low flip angle should also be relatively more immune to MT and $\mathrm{T}_{2}$ effects, than SSFP techniques, like MOLLI and shMOLLI. But even though MOLLI and shMOLLI may not be the most accurate techniques for $\mathrm{T}_{1}$ quantification, they have been found to be more precise than Look-Locker techniques.

\section{Water Exchange Effects}

For post-contrast measurements of $\mathrm{T}_{1}$ in myocardial tissue, the movement of water between spaces with and without contrast agent, can have important repercussions on the apparent $T_{1}$ of the tissue. Water exchange across the cell-membrane results in exchange of magnetization during an inversion recovery. Without any exchange of water across the transcytolemmal barrier, one would to a first approximation expect that after administration of an extracellular contrast agent, the inversion recovery curve resembles a sum of two exponentials: one representing the recovery in the extra-cellular space permeated by contrast, and a slower inversion recovery in the intra-cellular space not accessible to the contrast agent. In practice, myocardial inversion recovery curves appear to be well approximated by a single exponential, suggesting that the exchange of water across the transcytolemmal barrier spreads the effects of contrast-enhanced relaxation to the intra-cellular space. This effectively couples the relaxation recoveries in the two spaces, so that it is reasonably well approximated by a single-exponential.

The effect of water exchange on $T_{1}$ is often assumed to be fast enough that the $R_{1}$ in myocardial tissue increases linearly with the $\mathrm{R}_{1}$ in blood. But such a linear relationship only applies in the limit of fast transcytolemmal water exchange. The term "fast" here refers to the rate of water exchange relative to the $\mathrm{R}_{1}$ in the space permeated by contrast. When the fast exchange condition is no longer fulfilled, then water exchange becomes a bottle-neck for the relaxation recovery of the intra-cellular water, leading to a lower $\mathrm{R}_{1}$ of tissue, compared to the prediction for the fast-exchange limit. If the $\mathrm{R}_{1}$ data are interpreted by assuming fast exchange conditions, this can therefore lead to an underestimate of $E C V .{ }^{23}$ The effects of water-exchange in normal myocardial tissue become noticeable when $\mathrm{R}_{1}$ in blood $>\sim 3$ $\mathrm{s}^{-1}$. ${ }^{23}$ After a contrast injection, early measurements of $\mathrm{T}_{1}$ are therefore more likely to lead to a bias to underestimate $\mathrm{ECV}$, if one interprets the data under the assumption of fast water exchange. It may be then concluded that contrast in blood and tissue are not yet at 
equilibrium, though the source of error is due to an oversimplified interpretation of the $T_{1}$ measurements.

The consideration of the effects of water exchange is not only relevant for avoiding a bias in the estimation of ECV, but may also be used to extract additional useful information about the tissue structure. The rate of water exchange relates to the average lifetime of a water molecule within a cell. ${ }^{28}$ This so-called intra-cellular lifetime, can be related to the geometry of the cell, because it is proportional to the volume-to-surface area ratio (V/S) of the cells. In the case of cardiomyocytes with a length-to-diameter ration of approximately $4: 1, \mathrm{~V} / \mathrm{S}$ is to a first approximation equal to the cell-diameter, which changes with the development of cellhypertrophy. Through quantification of the intra-cellular lifetime, by post-contrast $T_{1}$ measurements over a relatively wide range of contrast concentrations, it is feasible to detect cardiomyocyte hypertrophy. ${ }^{29}$

\section{Current Limitations}

For cardiac applications, the requirement to acquire longitudinal magnetization-recovery data within a breath-hold, continues to be an important bottle-neck that constrains $\mathrm{T}_{1}$ protocols. For this reason, almost all $\mathrm{T}_{1}$-mapping sequences in current use rely on $2 \mathrm{D}$ image acquisitions. Breath-hold acquisitions are repeated to cover the entire heart, e.g. with a stack of short axis slices. With the need to perform $\mathrm{T}_{1}$-mapping both before and after contrast administration, this procedure can become cumbersome, and a single $3 \mathrm{D} \mathrm{T}_{1}$-mapping acquisition would be preferable. To achieve $3 \mathrm{D}_{1}$ mapping will require highly accelerated parallel imaging, and sparse sampling. An important advantage of 3D imaging would be that slice "inflow effects" for the blood signal could be reduced. With Look-Locker acquisitions the inflow effects result in cardiac-cycle dependent modulation of the signal, which grows in prominence as one increases the RF flip angle for the image read-out.

\section{Conclusions}

Interest in $\mathrm{T}_{1}$-mapping of the heart has increased markedly over the last few years due to new applications which address the need to detect changes in tissue, such as expansion of the extra-cellular space, development of cell hypertrophy, and increases in the free water content. Look-Locker, and modified Look-Locker (MOLLI, shMOLLI, SASHA) techniques have emerged as the mainstays for $\mathrm{T}_{1}$ mapping of the heart. Mechanisms that may affect the longitudinal magnetization recoveries measured with each of these techniques (like magnetization transfer effects) are still being investigated. While it can be said that the currently used cardiac $T_{1}$ mapping techniques are relatively precise, their accuracy, and potential sources of biasing effects on $\mathrm{T}_{1}$, remain an area of active investigation. Notwithstanding such open questions, $\mathrm{T}_{1}$ mapping has proven highly valuable to improve the already excellent capabilities of CMR for myocardial tissue characterization.

\section{Acknowledgements}

The authors gratefully acknowledge support of research related to the topic of this review article through awards R01HL090634 and R01HL091157 from the National Heart, Lung and Blood (NHLBI). The authors express their gratitude to Dr. Rob van der Geest (University of Leiden, The Netherlands) for useful discussions regarding $\mathrm{T}_{1}$

Top Magn Reson Imaging. Author manuscript; available in PMC 2017 February 14. 
mapping, and to Dr. Michael Deimling (Siemens Medical Systems, Erlangen, Germany) for sharing over the years his expertise in pulse sequence techniques for $\mathrm{T}_{1}$ quantification.

\section{References}

1. Sparrow P, Messroghli DR, Reid S, Ridgway JP, Bainbridge G, Sivananthan MU. Myocardial T1 mapping for detection of left ventricular myocardial fibrosis in chronic aortic regurgitation: pilot study. American Journal of Roentgenology. 2006; 187(6):W630-635. [PubMed: 17114517]

2. Borer JS, Truter S, Herrold EM, Falcone DJ, Pena M, Carter JN, Dumlao TF, Lee JA, Supino PG. Myocardial fibrosis in chronic aortic regurgitation: molecular and cellular responses to volume overload. Circulation. 2002; 105(15):1837-1842. [PubMed: 11956128]

3. Liu SK, Magid NR, Fox PR, Goldfine SM, Borer JS. Fibrosis, myocyte degeneration and heart failure in chronic experimental aortic regurgitation. Cardiology. 1998; 90(2):101-109. [PubMed: 9778546]

4. Jerosch-Herold M, Sheridan DC, Kushner JD, Nauman D, Burgess D, Dutton D, Alharethi R, Li DX, Hershberger RE. Cardiac magnetic resonance imaging of myocardial contrast uptake and blood flow in patients affected with idiopathic or familial dilated cardiomyopathy. American Journal of Physiology-Heart and Circulatory Physiology. 2008; 295(3):H1234-H1242. [PubMed: 18660445]

5. Flett AS, Hayward MP, Ashworth MT, Hansen MS, Taylor AM, Elliott PM, McGregor C, Moon JC. Equilibrium contrast cardiovascular magnetic resonance for the measurement of diffuse myocardial fibrosis: preliminary validation in humans. Circulation. 2010; 122(2):138-144. [PubMed: 20585010]

6. Flett AS, Sado DM, Quarta G, Mirabel M, Pellerin D, Herrey AS, Hausenloy DJ, Ariti C, Yap J, Kolvekar S, Taylor AM, Moon JC. Diffuse myocardial fibrosis in severe aortic stenosis: an equilibrium contrast cardiovascular magnetic resonance study. Eur Heart J Cardiovasc Imaging. 2012

7. Ferreira VM, Piechnik SK, Dall'Armellina E, Karamitsos TD, Francis JM, Choudhury RP, Friedrich MG, Robson MD, Neubauer S. Non-contrast T1-mapping detects acute myocardial edema with high diagnostic accuracy: a comparison to T2-weighted cardiovascular magnetic resonance. J Cardiovasc Magn Reson. 2012; 14:42. [PubMed: 22720998]

8. Dall'Armellina E, Ferreira VM, Kharbanda RK, Prendergast B, Piechnik SK, Robson MD, Jones M, Francis JM, Choudhury RP, Neubauer S. Diagnostic value of pre-contrast T1 mapping in acute and chronic myocardial infarction. JACC Cardiovasc Imaging. 2013; 6(6):739-742. [PubMed: 23764100]

9. Dass S, Suttie JJ, Piechnik SK, Ferreira VM, Holloway CJ, Banerjee R, Mahmod M, Cochlin L, Karamitsos TD, Robson MD, Watkins H, Neubauer S. Myocardial tissue characterization using magnetic resonance noncontrast T1 mapping in hypertrophic and dilated cardiomyopathy. Circ Cardiovasc Imaging. 2012; 5(6):726-733. [PubMed: 23071146]

10. Look D, Locker D. Time saving in measurement of NMR and EPR relaxation times. Rev. Sci. Instrum. 1970; 41:250-251.

11. Crawley AP, Henkelman RM. A comparison of one-shot and recovery methods in T1 imaging. Magn. Res. Med. 1988; 7:23-34.

12. Kay I, Henkelman RM. Practical implementation and optimization of one-shot T1 imaging. Magn Reson Med. 1991; 22(2):414-424. [PubMed: 1812376]

13. Brix G, Schad LR, Deimling M, Lorenz WJ. Fast and precise T1 imaging using a TOMROP sequence. Magn. Res. Imag. 1990; 8:351-356.

14. Gowland PA, Leach MO. Fast and accurate measurements of T1 using a multi-readout single inversion-recovery sequence. Magn Reson Med. 1992; 26(1):79-88. [PubMed: 1625570]

15. Scheffler K, Hennig J. T1 quantification with inversion recovery TrueFISP. Magn Reson Med. 2001; 45(4):720-723. [PubMed: 11284003]

16. Messroghli DR, Greiser A, Frohlich M, Dietz R, Schulz-Menger J. Optimization and validation of a fully-integrated pulse sequence for modified look-locker inversion-recovery (MOLLI) T1 mapping of the heart. J Magn Reson Imaging. 2007; 26(4):1081-1086. [PubMed: 17896383] 
17. Messroghli DR, Radjenovic A, Kozerke S, Higgins DM, Sivananthan MU, Ridgway JP. Modified Look-Locker inversion recovery (MOLLI) for high-resolution T1 mapping of the heart. Magn Reson Med. 2004; 52(1):141-146. [PubMed: 15236377]

18. Piechnik SK, Ferreira VM, Dall'Armellina E, Cochlin LE, Greiser A, Neubauer S, Robson MD. Shortened Modified Look-Locker Inversion recovery (ShMOLLI) for clinical myocardial T1mapping at 1.5 and $3 \mathrm{~T}$ within a 9 heartbeat breathhold. J Cardiovasc Magn Reson. 2010; 12:69. [PubMed: 21092095]

19. Chow K, Flewitt JA, Green JD, Pagano JJ, Friedrich MG, Thompson RB. Saturation recovery single-shot acquisition (SASHA) for myocardial T mapping. Magn Reson Med. 2013

20. Pickup S, Wood AK, Kundel HL. A novel method for analysis of TOMROP data. J Magn Reson Imaging. 2004; 19(4):508-512. [PubMed: 15065176]

21. Deichmann R, Haase A. Quantification of T1 values by SNAPSHOT-FLASH NMR imaging. J. Magn. Reson. 1992; 96:608-612.

22. Messroghli DR, Rudolph A, Abdel-Aty H, Wassmuth R, Kuhne T, Dietz R, Schulz-Menger J. An open-source software tool for the generation of relaxation time maps in magnetic resonance imaging. BMC Med Imaging. 2010; 10:16. [PubMed: 20673350]

23. Coelho-Filho OR, Mongeon FP, Mitchell R, Moreno H Jr. Nadruz W Jr. Kwong R, Jerosch-Herold $M$. Role of transcytolemmal water-exchange in magnetic resonance measurements of diffuse myocardial fibrosis in hypertensive heart disease. Circ Cardiovasc Imaging. 2013; 6(1):134-141. [PubMed: 23159497]

24. Schmitt P, Griswold MA, Jakob PM, Kotas M, Gulani V, Flentje M, Haase A. Inversion recovery TrueFISP: quantification of T(1), T(2), and spin density. Magn Reson Med. 2004; 51(4):661-667. [PubMed: 15065237]

25. Robson MD, Piechnik SK, Tunnicliffe EM, Neubauer S. T1 measurements in the human myocardium: The effects of magnetization transfer on the SASHA and MOLLI sequences. Magn Reson Med. 2013

26. Kellman P, Herzka DA, Arai AE, Hansen MS. Influence of Off-resonance in myocardial T1mapping using SSFP based MOLLI method. J Cardiovasc Magn Reson. 2013; 15:63. [PubMed: 23875774]

27. Nacif MS, Turkbey EB, Gai N, Nazarian S, van der Geest RJ, Noureldin RA, Sibley CT, Ugander M, Liu S, Arai AE, Lima JA, Bluemke DA. Myocardial T1 mapping with MRI: comparison of Look-Locker and MOLLI sequences. J Magn Reson Imaging. 2011; 34(6):1367-1373. [PubMed: 21954119]

28. Landis CS, Li X, Telang FW, Molina PE, Palyka I, Vetek G, Springer CS Jr. Equilibrium transcytolemmal water-exchange kinetics in skeletal muscle in vivo. Magn Reson Med. 1999; 42(3):467-478. [PubMed: 10467291]

29. Coelho-Filho OR, Shah RV, Mitchell R, Neilan TG, Moreno H Jr. Simonson B, Kwong R, Rosenzweig A, Das S, Jerosch-Herold M. Quantification of cardiomyocyte hypertrophy by cardiac magnetic resonance: implications for early cardiac remodeling. Circulation. 2013; 128(11):12251233. [PubMed: 23912910] 


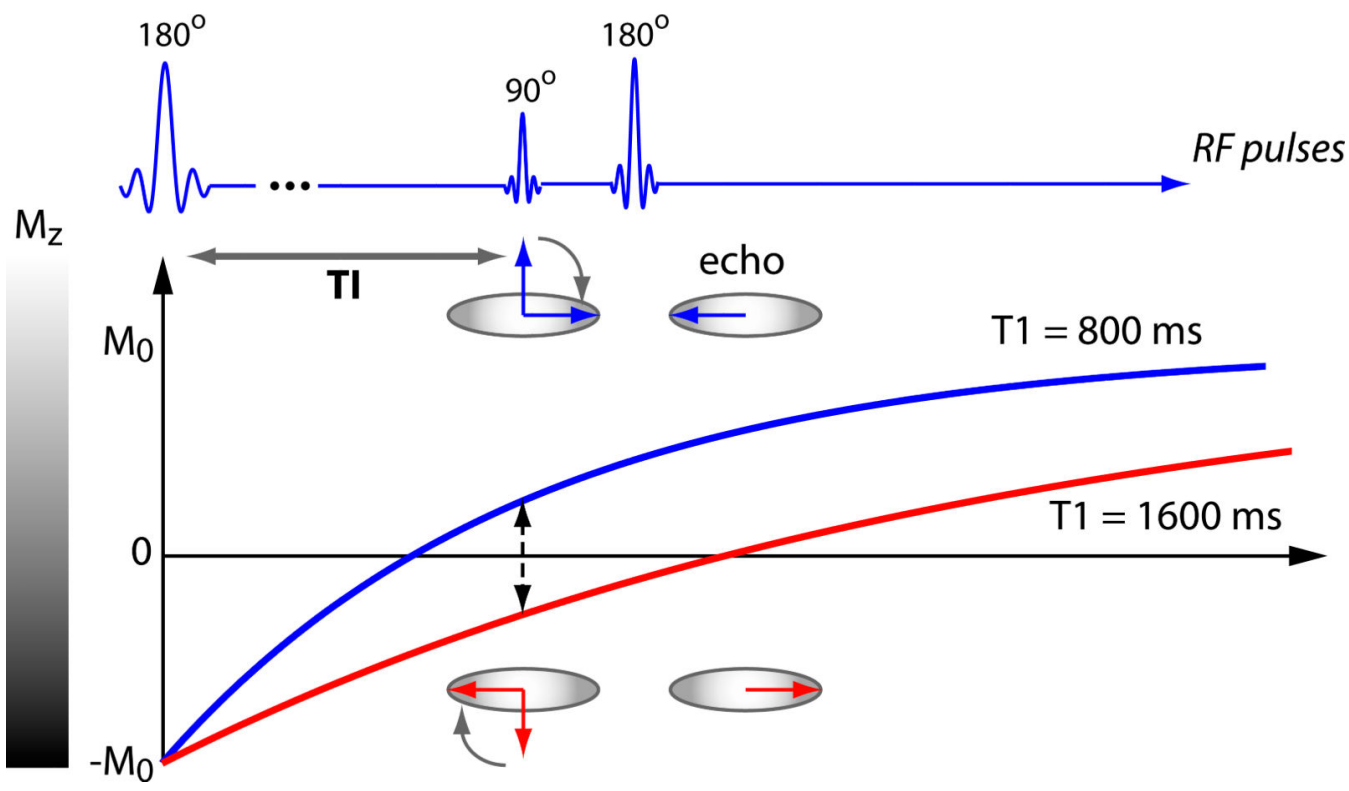

Figure 1.

Inversion-recovery measurement, consisting of an inversion pulse, followed some time after inversion (TI) by a $90^{\circ}-180^{\circ}$ pulse pair to produce a spin-echo with an amplitude proportional to the longitudinal magnetization at time TI. With phase-sensitive signal detection it is feasible to preserve the polarity of the longitudinal magnetization at time TI in the spin-echo signal. This inversion-recovery spin-echo sequence has to be repeated for a range of TI values to sample the inversion recovery at multiple time points and quantify $\mathrm{T}_{1}$. Before each inversion pulse, the magnetization should be in its equilibrium state, meaning that between repetitions of the inversion recovery measurement a time equivalent to $4-5 \times \mathrm{T}_{1}$ should elapse. 

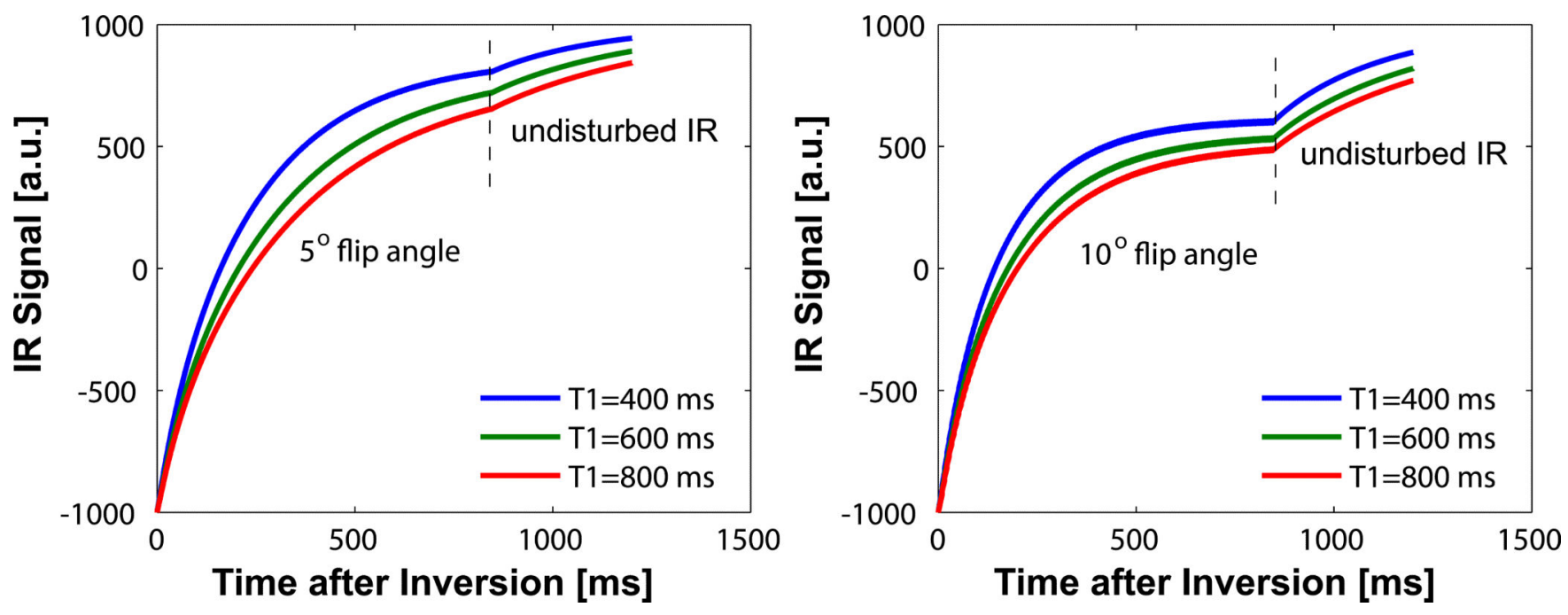

Figure 2.

Inversion recovery curves (in arbitrary units [a.u.]) during a Look-Locker experiment, with a radio-frequency (RF) pluse train with flip angle of $5^{\circ}$ in (a) and $10^{\circ}$ in (b). The inversion recovery is modified by the application of the RF pulse train, more so when the flip angle is higher, resulting in an effective $T_{1}$ that becomes increasingly shorter as the flip angle is increased. The $\mathrm{T}_{1} *$ measured with a Look-Locker type pulse sequence can be determined with a three-parameter fit to the expression $A+B * \exp \left(-T I / T_{1}\right)$, and the true $T_{1}$ is then calculated from these parameters, using equation 6. 


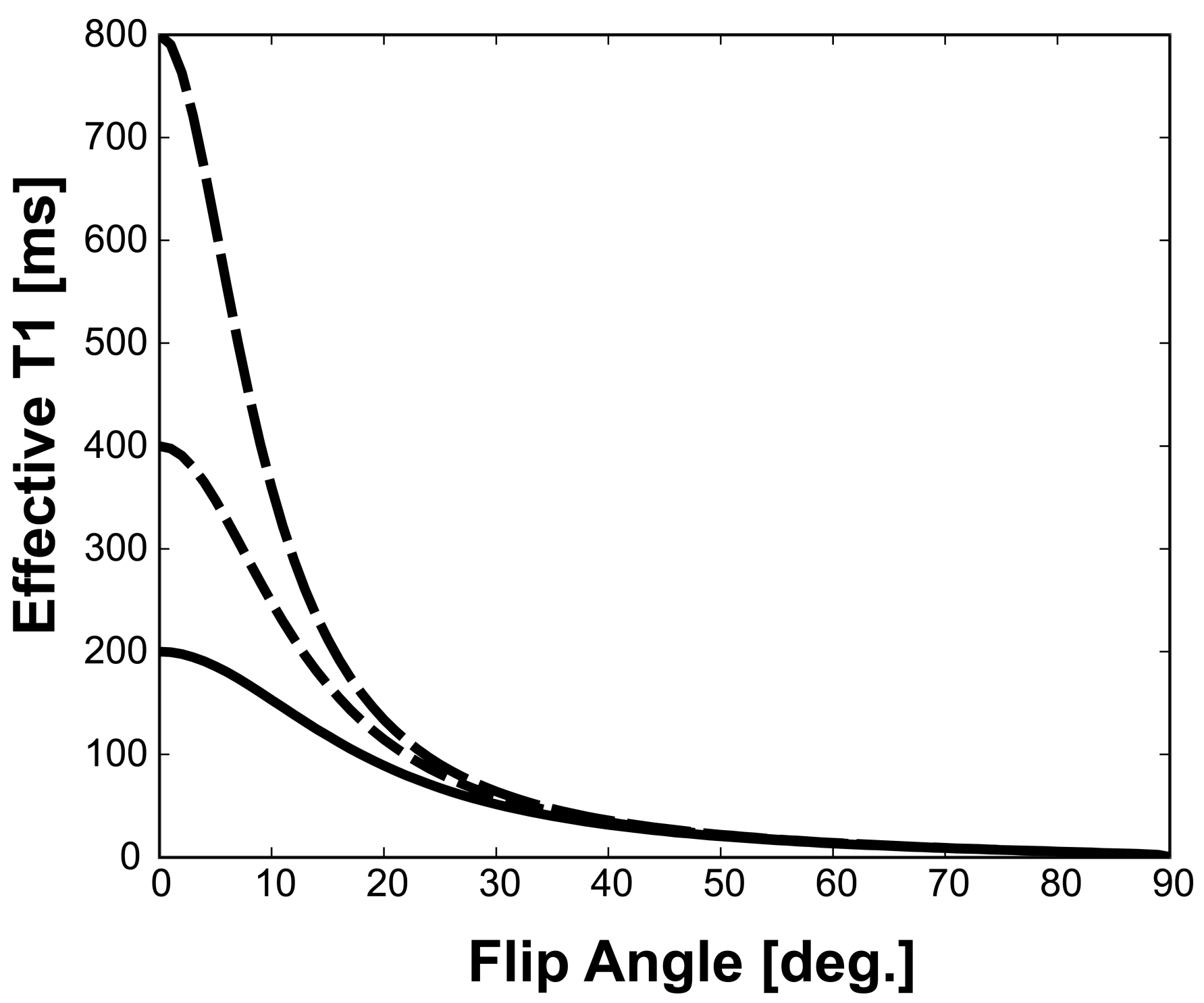

Figure 3.

The effective $\mathrm{T}_{1}$ measured with a Look-Locker type pulse sequence decreases with increasing flip angle, as shown here for three cases where the undisturbed inversion recovery (i.e. the $\mathrm{T}_{1}$ measured with a two-pulse experiment) would be $\mathrm{T}_{1}=800$ (long dashes), 400 (short dashes), and $200 \mathrm{~ms}$ (solid line). The repetition time for the RF pulse train was $10 \mathrm{~ms}$. It is apparent that with a TR on this order, one should use a $\mathrm{RF}$ flip angle $<\sim 20^{\circ}$ to maintain adequate sensitivity to $T_{1}$. 


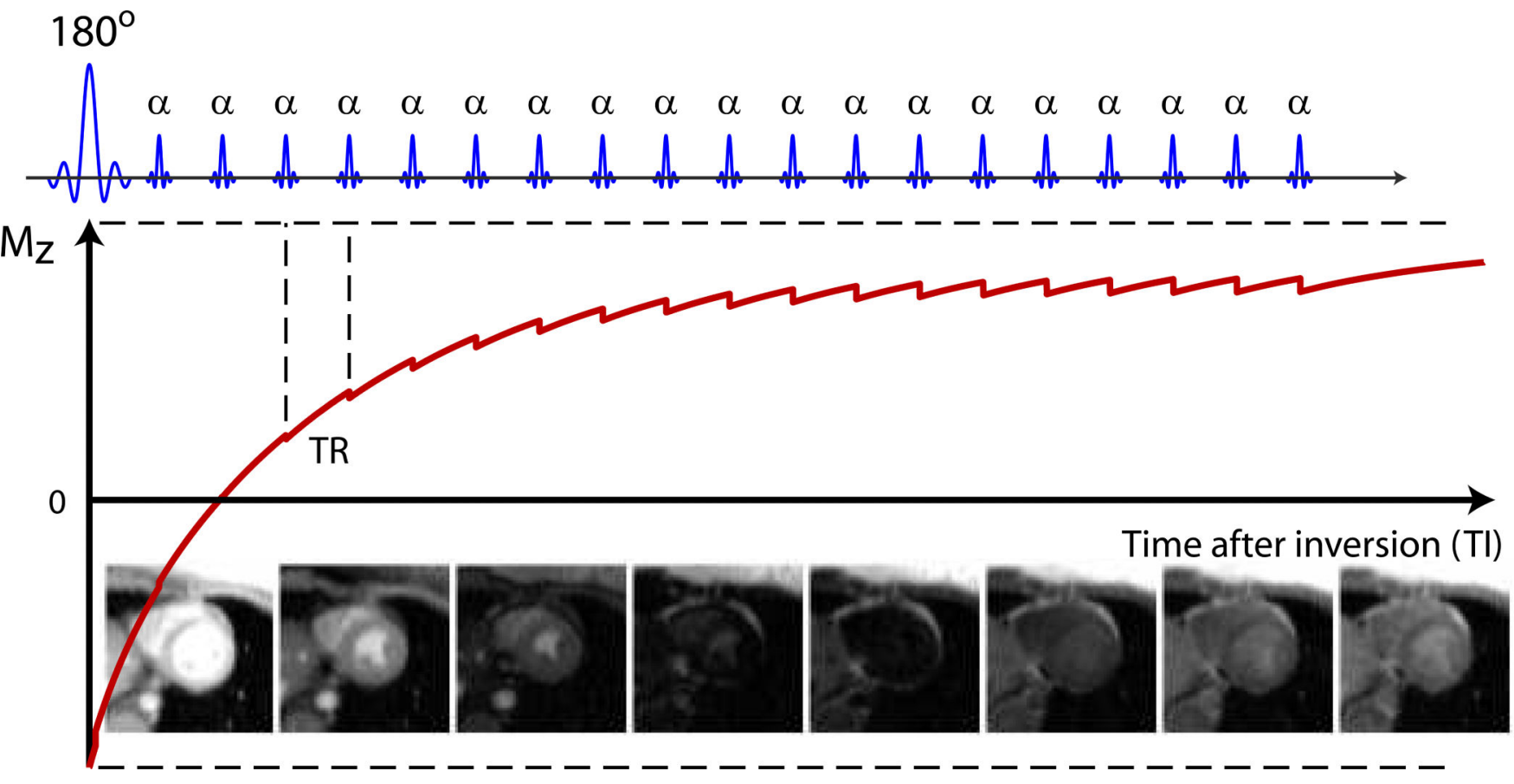

Figure 4.

The diagram illustrates the basic building block of a Look-Locker image acquisition, consisting of an inversion pulse, followed by a train of low-flip angle radio-frequency pulses (flip angle denoted by a). Not shown are the gradient pulses for slice-selection, phaseencoding, and gradient-echo read-out that are typically applied after each a-pulse. These gradient pulses do not disturb the inversion recovery. The basic building block in the diagram represents a single Look-Locker cycle with 20 different signal samples, corresponding to TI times, with increments that equal TR, the time between RF pulses. The LL-cycle is repeated $n$ number of times, where $n$ represents e.g. the number of phaseencoding steps. A subset of images in the figure corresponding to different TI times is shown in the lower inset. 


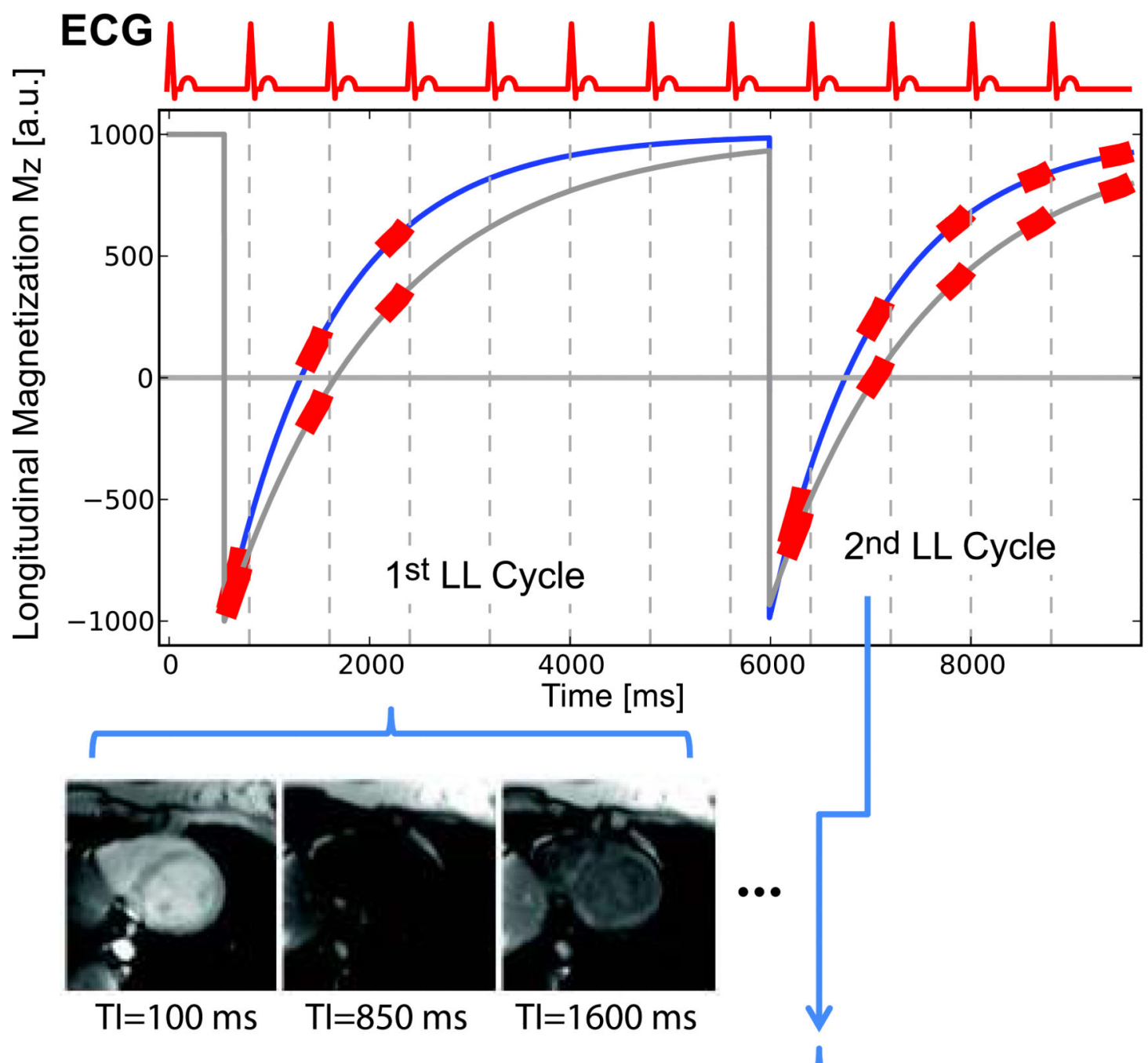

$\mathrm{Tl}=100 \mathrm{~ms}$

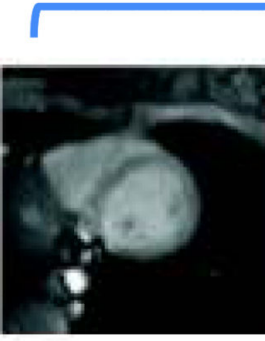

$\mathrm{Tl}=180 \mathrm{~ms}$

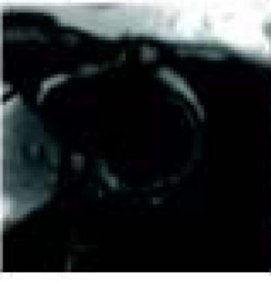

$\mathrm{Tl}=930 \mathrm{~ms}$

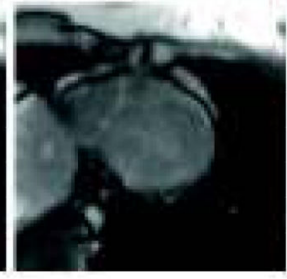

$\mathrm{Tl}=1680 \mathrm{~ms}$

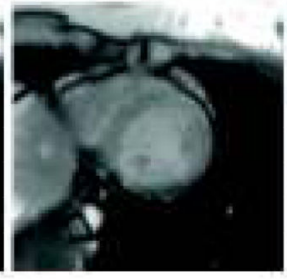

$\mathrm{TI}=2430 \mathrm{~ms}$

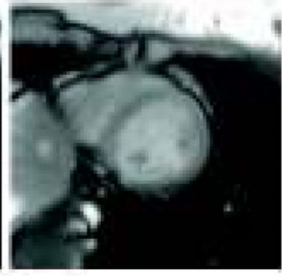

$\mathrm{Tl}=3180 \mathrm{~ms}$

Figure 5.

Acquisition scheme for modified Look-Locker (MOLLI) pulse sequence. The acquisition scheme in this example comprises two Look-Locker cycles, each initiated by an inversion pulse. The assumed heart rate was $80 \mathrm{bpm}$, and its duration $750 \mathrm{~ms}$. The blue line shows the evolution of the longitudinal magnetization over the course of the acquisition, assuming a $\mathrm{T}_{1}$ of $1100 \mathrm{~ms}$ (e.g. myocardium). The grey line shows the longitudinal magnetization for an assumed $\mathrm{T}_{1}$ of $1600 \mathrm{~ms}$ (e.g. blood). The grey rectangles overlaid on the signal curves denote the times where images are acquired in single-shot mode $(100 \mathrm{~ms})$ with steady-state free precession (SSFP). By virtue of the SSFP acquisition the signal evolution is not 
disturbed under ideal conditions during the image read-out. The vertical dotted lines denote the ECG R-wave occurrence, and the red-trace on top shows a simulated ECG trace. During the first Look-Locker cycle the first TI value has the shortest value of $100 \mathrm{~ms}$, which is incremented in this case by $80 \mathrm{~ms}$ for the next LL cycles. After the first LL cycle, the sequence is paused for 2 additional heart cycles to allow full, and undisturbed signal recovery, before the next inversion pulse is applied. Note that all images are acquired for the same cardiac phase, yet different times after inversion. 


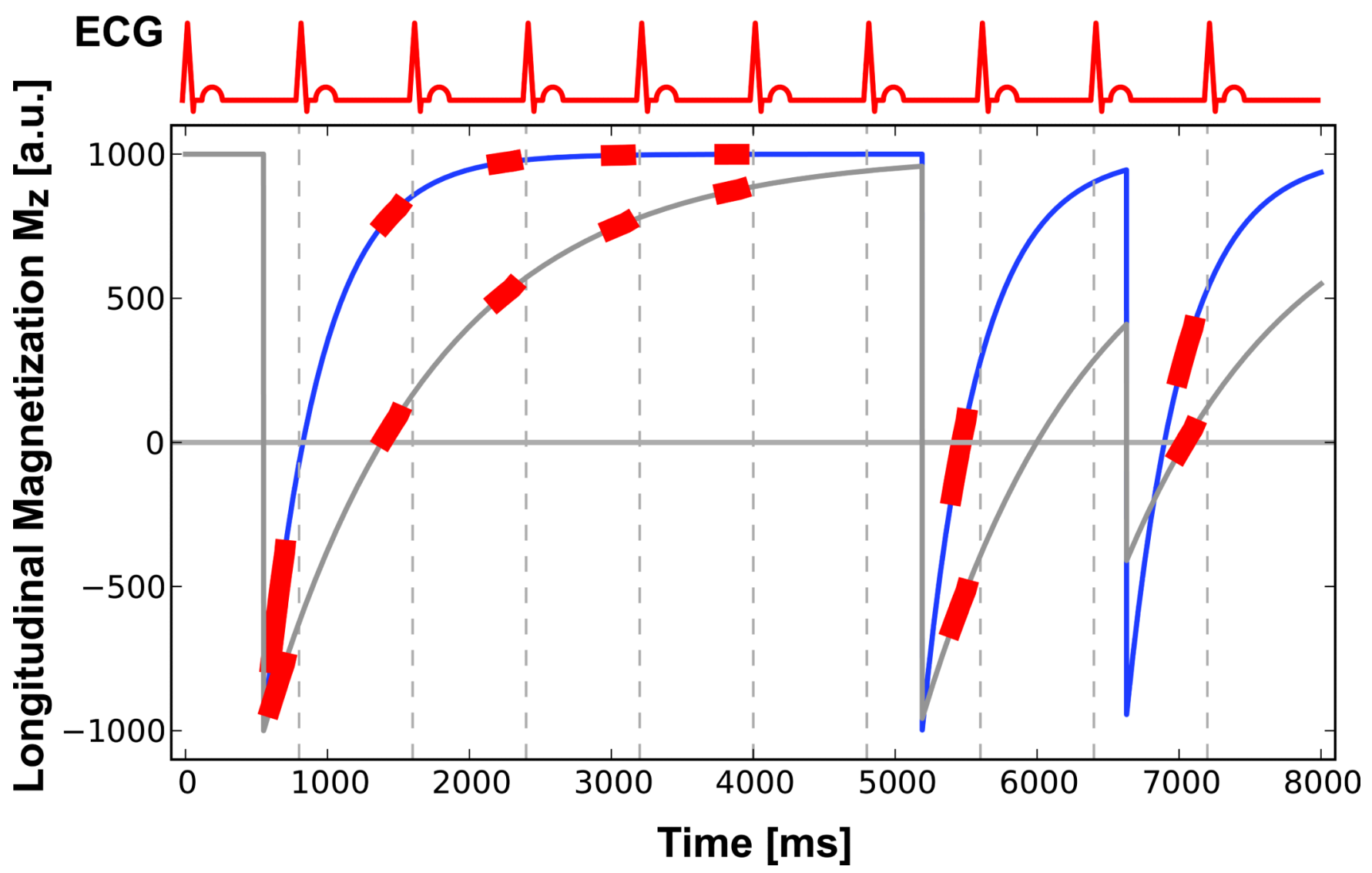

Figure 6.

Pulse sequence scheme for shortened modified Look-Locker (shMOLLI) acquisition. This variant of MOLLI uses three Look-Locker (LL) cycles, with the last two shortened to reduce the breath-hold duration. The grey and blue lines show the evolution of the longitudinal magnetization of myocardial scar and normal myocardium over the course of the acquisition, assuming a $\mathrm{T}_{1}$ of $400 \mathrm{~ms}$, and $1200 \mathrm{~ms}$, respectively. For the longer $\mathrm{T}_{1}(1200$ $\mathrm{ms}$ ), the recovery after the first Look-Locker cycle is incomplete, but for longer $\mathrm{T}_{1}$ values the additional signal samples with short TI's can be discarded with a relatively minor effects on $\mathrm{T}_{1}$ accuracy. Therefore with shMOLLI a conditional $\mathrm{T}_{1}$ quantification scheme is used, where the signal samples for the last two LL cycles, or just the last LL cycle, are left out depending on heuristic criteria that minimize the fit error, and also take into account whether $\mathrm{T}_{1}$ is longer than the heart beat interval or not. Other details of this figure follow the same scheme as in Figure 5. 


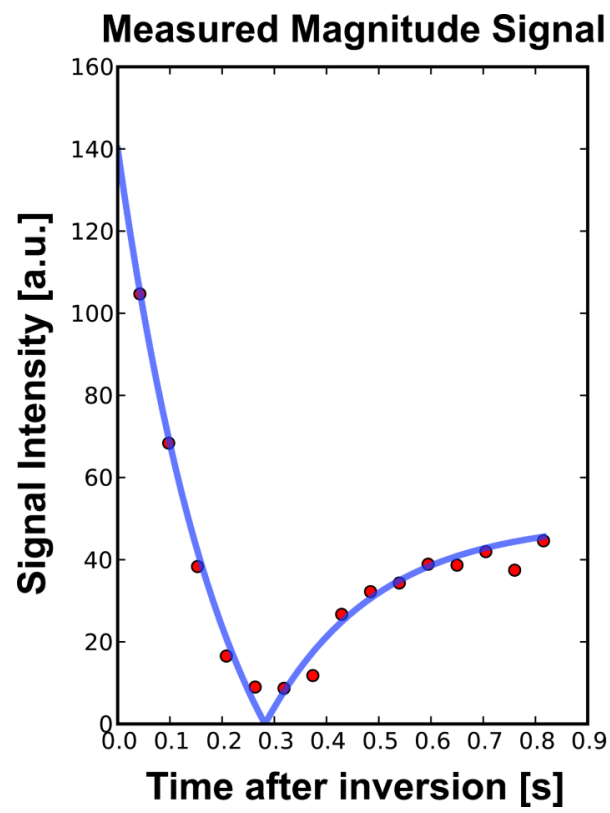

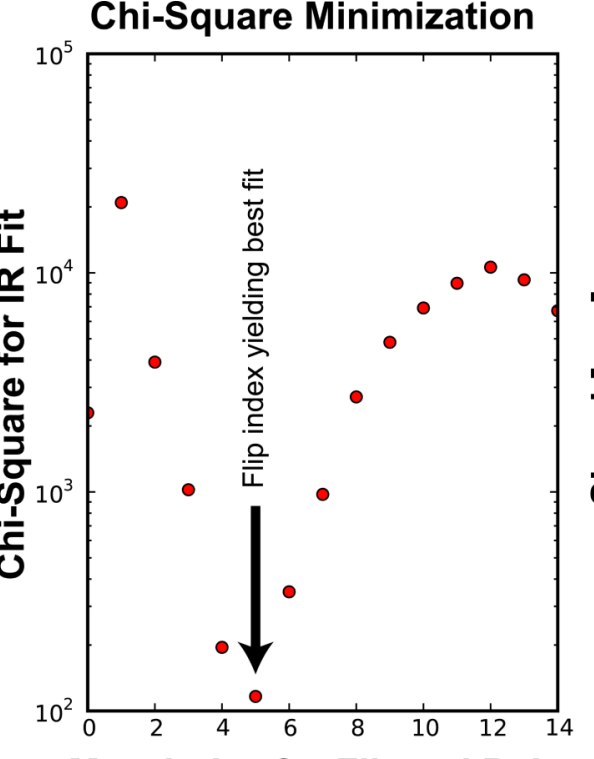

Max. index for Flipped Points

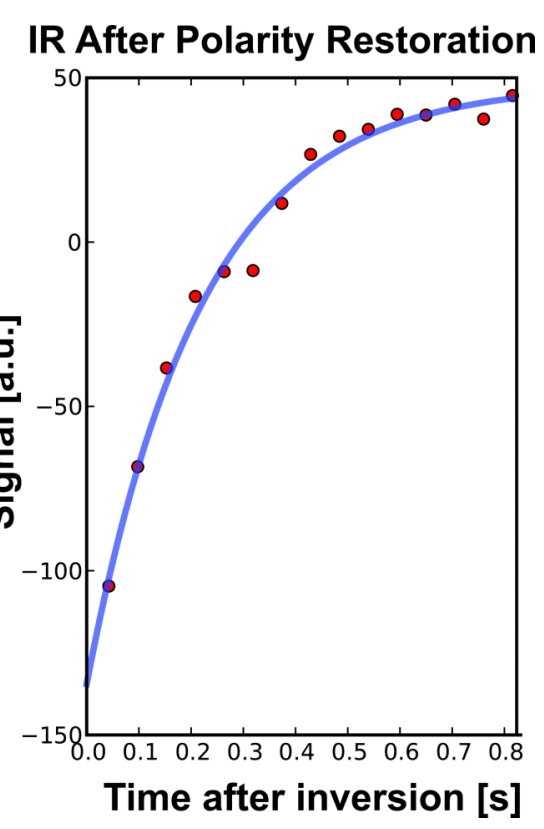

Figure 7.

Left: Inversion-recovery curves are typically extracted from magnitude images, resulting in inversion recovery curves that have a V-like shape. Before fitting the curves to the equation for an inversion recovery, one can restore the polarity in the signal intensity curves. This is particularly useful if the inversion recovery is only sampled for few TI's, as the loss of signal polarity then renders it more challenging to fit the magnitude data. Middle: Signal polarity restoration can be done iteratively, by inverting the data points up to the $n^{\prime}$ th sample. For each iteration the resulting signal curve is fit, and the agreement with the measured data is measured by the sum of the squared distances between model curve and data points (chisquare). Next, chi-square is plotted as a function of the flip point number $n$, and the optimal flip point location is chosen from the minimum of chi-square curve. Right: After restoration of the signal polarity, the signal intensity curve can be fit to equation 4 in the text. The solid lines in the left and right panel represent the model fit.

Top Magn Reson Imaging. Author manuscript; available in PMC 2017 February 14. 


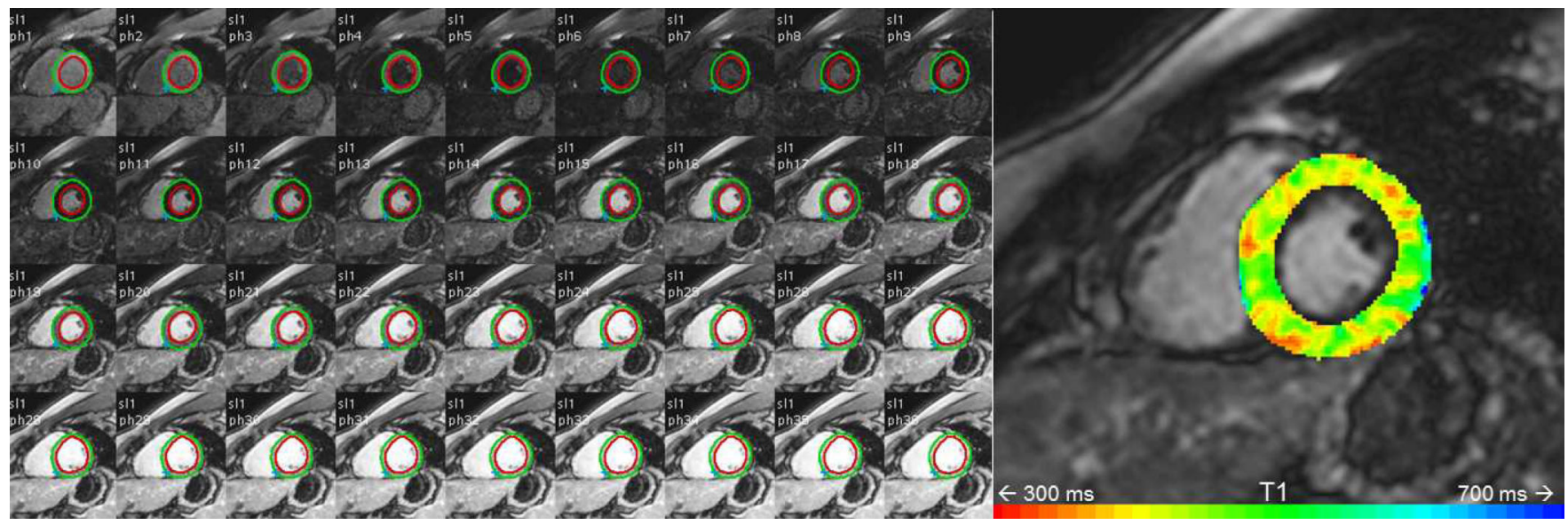

Figure 8.

Left: Series of images with varying inversion times acquired using a Look-Locker sequence throughout one cardiac cycle. Right: Pixel-wise $\mathrm{T}_{1}$ map derived from the data. To obtain pixel-wise intensity curves that are corrected for cardiac motion, image registration is required to find point/tissue correspondence between the imaging frames. This is realized in this example by manually outlining the endocardial and epicardial contours in four key frames followed by automated contour detection to find the contours in the other frames. The relative position of a pixel in the myocardium can be described by its radial position, i.e. the relative distance to the two defined boundaries, and the circumferential location assessed by the angular position relative to the posterior junction of the RV with the LV (indicated as blue maker in the left panel). (Images provided by Dr. Rob van der Geest, University of Leiden, Netherlands) 


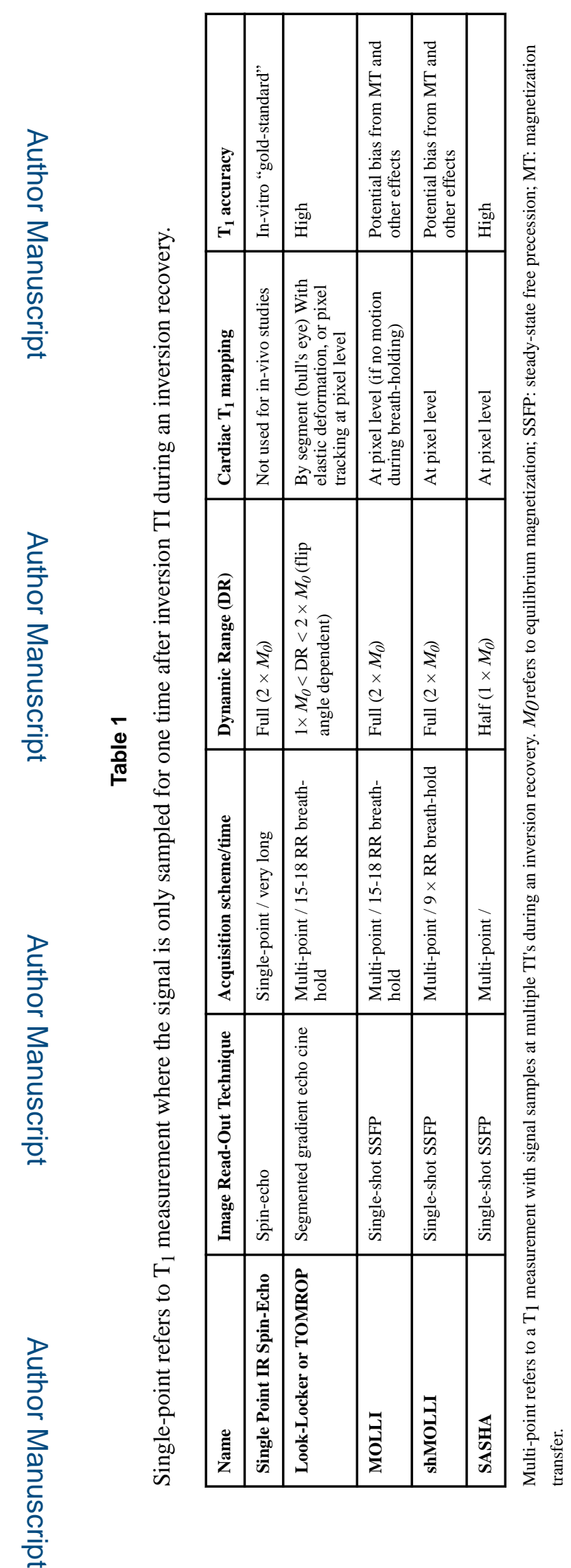

Top Magn Reson Imaging. Author manuscript; available in PMC 2017 February 14. 